\title{
ASYMPTOTIC BEHAVIOR OF SOLUTIONS IN LINEAR 2- OR 3-D THERMOELASTICITY WITH SECOND SOUND
}

\author{
BY \\ REINHARD RACKE \\ Department of Mathematics and Statistics, University of Konstanz, 78457 Konstanz, Germany
}

\begin{abstract}
We consider thermoelastic systems in two or three space dimensions where thermal disturbances are modeled propagating as wavelike pulses traveling at finite speed. This is done using Cattaneo's law for heat conduction instead of Fourier's law. For Dirichlet type boundary conditions, the exponential stability of the now purely, but slightly damped, hyperbolic system is proved in the radially symmetric case.
\end{abstract}

1. Introduction. We consider two- or three-dimensional thermoelastic bodies including the second sound effect. The latter turns the classical thermoelastic system of hyperbolic-parabolic coupled type into a purely hyperbolic, but damped hyperbolic one. This way the paradox of infinite propagation speed of heat pulses is overcome, which is regarded as being important in some applications like pulsed laser heating of solids; compare $[11,18]$. The governing differential equations for the displacement vector $u=u(t, x) \in \mathbb{R}^{n}, n=2$ or 3 , the temperature difference $\theta=\theta(t, x)$, and the heat flux vector $q=q(t, x) \in \mathbb{R}^{n}$, where $t \geq 0$ and $x \in \Omega \subset \mathbb{R}^{4}, \Omega$ denoting the bounded reference configuration with smooth boundary $\partial \Omega\left(C^{2}\right.$ is sufficient), are the following (compare $[2,10,14,16])$ for a homogeneous isotropic medium:

$$
\begin{gathered}
u_{t t}-\mu \Delta u-(\mu+\lambda) \nabla \operatorname{div} u+\beta \nabla \theta=0, \\
\theta_{t}+\gamma \operatorname{div} q+\delta \operatorname{div} u_{t}=0, \\
\tau_{0} q_{t}+q+\kappa \nabla \theta=0 .
\end{gathered}
$$

These equations are completed by initial conditions

$$
u(0, \cdot)=u_{0}, u_{t}(0, \cdot)=u_{1}, \theta(0, \cdot)=\theta_{0}, q(0, \cdot)=q_{0}
$$

and the following boundary conditions for a rigidly clamped body held at constant temperature on the boundary:

$$
u(t, \cdot)=0, \theta(t, \cdot)=0 \quad \text { on } \partial \Omega, t \geq 0 .
$$

Received June 13, 2001.

2000 Mathematics Subject Classification. Primary 74F05, 74H40.

E-mail address: reinhard.racke@uni-konstanz.de 
The parameters $\mu, \beta, \gamma, \delta, \tau_{0}$ and $\kappa$ are positive constants. $\lambda+2 \mu>0$, where $\mu$ and $\lambda$ represent the Lamé moduli and $\tau_{0}$ is the relaxation time, a small parameter compared to the others. For $\tau_{0}=0$ we return to classical thermoelasticity since in this case Cattaneo's law for heat conduction, cxpressed in (1.3) and responsible for the overall hyperbolic character of the system, turns into Fourier's law

$$
q=-\kappa \nabla \theta
$$

making (1.2), (1.3) a parabolic equation for the temperature:

$$
\theta_{t}-\gamma \kappa \Delta \theta+\delta \operatorname{div} u_{t}=0 \text {. }
$$

It is known for classical thermoclasticity (1.1), (1.6) that in general bounded domains, for instance in all domains that allow reflecting rays, there is no uniform decay rate for the energy; see $[6,7,5]$. On the other hand, it has been proved that whenever the rotation $\operatorname{rot} u$ vanishes identically - for instance in a radially symmetric domain $\Omega$ with radially symmetric data-then the energy decays exponentially; see $[4,5]$. Now back to thermoelasticity with second sound (1.1)-(1.3). What can one expect for this even less dissipative - while only damped--hyperbolic system? Will the dissipation which is still given through heat conduction be strong enough at least in radially symmetric situations to exponentially stabilize all components $u, \theta$, and $q$ ? We recall that in [13] the corresponding question was answered in the affirmative even for nonlinear systems. Here we shall prove the same for the radially symmetric case after a discussion of the general asymptotic behavior in terms of possible oscillatory behavior.

There seems to be no result on the precise decay up to now. In [15] the stability of the null solution was investigated; compare also [17] in one dimension. Thus we present the first description of the exponential stability. For the proof, appropriate Lyapunov functions have to be found, combining techniques from classical thermoelasticity [4] and from one-dimensional situations [13].

We remark that corresponding nonlinear problems are also of interest for the applications, and are under investigation. Another class of problems concerns Cauchy problems, i.e., $\Omega=\mathbb{R}^{m}, m=1,2,3$.

In Sec. 2 we look at the well-posedness in suitable Sobolev spaces that will allow a clear description of the generic asymptotic behavior; in particular, the connection to classical thermoelasticity will become apparent. In Sec. 3 the exponential decay will be proved for systems where $\operatorname{rot} u=0=\operatorname{rot} q$, which has its application in Sec. 4 for radially symmetric situations.

2. Well-posedness and remarks on general asymptotics. We transform the initial boundary value problem (1.1)-(1.5) for $(u, \theta, q)$ to a first-order system for

$$
V \equiv\left(\begin{array}{c}
V^{1} \\
V^{2} \\
V^{3} \\
V^{4}
\end{array}\right):=\left(\begin{array}{c}
u \\
u_{t} \\
\theta \\
q
\end{array}\right) \in \mathbb{R}^{3 n+1}, \quad V(0) \equiv V_{0}:=\left(\begin{array}{c}
u_{0} \\
u_{1} \\
\theta_{0} \\
q_{0}
\end{array}\right),
$$


observing that $(1.1)-(1.3)$ is equivalent to

$$
\begin{gathered}
k \delta u_{t t}+\kappa \delta E u+\kappa \delta \beta \nabla \theta=0, \\
\kappa \beta \theta_{t}+\kappa \beta \gamma \operatorname{div} q+\kappa \delta \beta \operatorname{div} u_{t}=0, \\
\beta \gamma \tau_{0} q_{t}+\beta \gamma q+\kappa \beta \gamma \nabla \theta=0,
\end{gathered}
$$

where

$$
E u:=-\mu \Delta u-(\mu+\lambda) \nabla \operatorname{div} u
$$

Let

$$
Q:=\left(\begin{array}{cccc}
1 & 0 & 0 & 0 \\
0 & \kappa \delta & 0 & 0 \\
0 & 0 & \kappa \beta & 0 \\
0 & 0 & 0 & \beta \gamma \tau_{0}
\end{array}\right), \quad N:=\left(\begin{array}{cccc}
0 & -1 & 0 & 0 \\
\kappa \delta E & 0 & \kappa \delta \beta \nabla & 0 \\
0 & \kappa \delta \beta \operatorname{div} & 0 & \kappa \beta \gamma \operatorname{div} \\
0 & 0 & \kappa \beta \gamma \nabla & \beta \gamma
\end{array}\right)
$$

in obvious notation, because " 0 " can denote a column or row vector in $\mathbb{R}^{n}$ with zero entries or the null matrix in $\mathbb{R}^{n^{2}}$.

Then $V$ satisfies

$$
V_{t}(t)+Q^{-1} N V(t)=0, \quad V(0)=V_{0} .
$$

Solving (2.6) in appropriate spaces is equivalent to solving (1.1)--(1.4) in appropriate spaces.

Let the operator $E$, formally defined in (2.5), be given by

$$
\begin{gathered}
E: D(E) \subset\left(L^{2}(\Omega)\right)^{n} \rightarrow\left(L^{2}(\Omega)\right)^{n}, \\
D(E):=\left(H^{2}(\Omega) \cap H_{0}^{1}(\Omega)\right)^{n}, \\
E u:=-\mu \Delta u-(\mu+\lambda) \nabla \operatorname{div} u .
\end{gathered}
$$

Then $E$ is positively definite and selfadjoint (see [8]), and we can define

$$
\mathcal{H}:=\left(H_{0}^{1}(\Omega)\right)^{n} \times\left(L^{2}(\Omega)\right)^{n} \times L^{2}(\Omega) \times\left(L^{2}(\Omega)\right)^{n}
$$

with inner product

$$
\langle V, W\rangle_{\mathcal{H}}:=\left\langle E^{1 / 2} V^{1}, E^{1 / 2} W^{1}\right\rangle+\sum_{j=2}^{4}\left\langle V^{j}, W^{j}\right\rangle,
$$

where $\langle\cdot, \cdot\rangle$ denotes the usual $L^{2}(\Omega)^{[n]}$ inner product and norm $\langle g, h\rangle=\int_{\Omega} g(x) \bar{h}(x) d x$ and $\|g\|$, respectively.

$$
\begin{gathered}
A: D(A) \subset \mathcal{H} \rightarrow \mathcal{H}, \\
D(A):=\left\{V \in \mathcal{H} \mid V^{1} \in\left(H^{2}(\Omega)\right)^{n}, \quad V^{2} \in\left(H_{0}^{1}(\Omega)\right)^{n}, \quad V^{3} \in H_{0}^{1}(\Omega), V^{4} \in \tilde{D}\right\},
\end{gathered}
$$

where

$$
\tilde{D}:=\left\{G \in\left(L^{2}(\Omega)\right)^{n} \mid \operatorname{div} G \in L^{2}(\Omega)\right\}
$$

and

Then (2.6) turns into

$$
A V:=Q^{-1} N V \quad \text { for } V \in D(A) .
$$

$$
V_{t}(t)+A V(t)=0, \quad V(0)=V_{0} \in D(A)
$$


Lemma 2.1. (i) $D(A)$ is dense in $\mathcal{H}$ and $-A$ is dissipative.

(ii) $0 \in \varrho(A)(\equiv$ resolvent set of $A)$.

Proof. $\quad$ (i) The density of $D(A)$ is obvious. Let $W \in D(A)$, then

$$
\operatorname{Re}\langle-A W, W\rangle_{\mathcal{H}}=-\beta \gamma \int_{\Omega}\left|W^{4}\right|^{2} d x \leq 0 .
$$

(ii) Let $W \in D(A)$ with $A W=0$. Then by (2.8) we conclude

$$
W^{4}=0 \text {. }
$$

$(A W)^{1}=0$ implies that

$$
W^{2}=0
$$

then $(A W)^{4}=0$ implies that

$$
\nabla W^{3}=0
$$

whence, since $W^{3} \in H_{0}^{1}(\Omega)$,

$$
W^{3}=0
$$

follows. Finally, we get from $(A W)^{2}=0$

$$
E W^{1}=0, \quad W^{1} \in D(E),
$$

implying

$$
W^{1}=0
$$

altogether: $W=0$, i.e., $A^{-1}$ exists.

Now we solve $A W=F$ for arbitrary $F \in \mathcal{H}$.

$$
A W=F
$$

is equivalent to

$$
\begin{gathered}
-W^{2}=F^{1}, \\
E W^{1}+\beta \nabla W^{3}=F^{3}, \\
\beta \operatorname{div} W^{2}+\gamma \operatorname{div} W^{4}=F^{3}, \\
\kappa \nabla W^{3}+W^{4}=\tau_{0} F^{4} .
\end{gathered}
$$

In view of (2.9) we define

$$
W^{2}:=-F^{1} \in H_{0}^{1}(\Omega)
$$

also yielding

$$
\left\|W^{2}\right\|=\left\|F^{1}\right\| \leq\|F\| .
$$

In view of (2.11), $W^{4}$ should satisfy

$$
\gamma \operatorname{div} W^{4}=F^{3}-\beta \operatorname{div} F^{1}
$$

or, looking at $(2.12), W^{3}$ should satisfy

$$
\gamma \kappa \Delta W^{3}=\gamma \tau_{0} \operatorname{div} F^{4}-F^{3}-\beta \operatorname{div} F^{1},
$$

where the right-hand side belongs to $H^{-1}(\Omega)$, which is the dual space of $H_{0}^{1}(\Omega)$.

For (2.15) there exists a unique solution $W^{3} \in H_{0}^{1}(\Omega)$ satisfying

$$
\left\|W^{3}\right\|+\left\|\nabla W^{3}\right\| \leq c\left(\left\|F^{4}\right\|+\left\|F^{3}\right\|+\left\|F^{1}\right\|\right) \leq c\|F\|,
$$


where $c$ here and in the sequel will denote a generic constant possibly varying from estimate to estimate. Now let

$$
W^{4}:=\tau_{0} F_{4}-\kappa \nabla W^{3}
$$

Then $W^{4} \in \tilde{D}$ and

$$
\left\|W^{4}\right\| \leq c\|F\|
$$

Finally, let $W^{1} \in D(E)$ be the unique solution to

$$
E W^{1}=-\beta \nabla W^{3}+F_{2}
$$

which satisfies

$$
\left\|E^{1 / 2} W^{1}\right\| \leq c\|F\|
$$

Then the construct $W=\left(W^{1}, W^{2}, W^{3}, W^{4}\right)^{\prime}$ satisfies $W \in D(A), A W=F$, and by (2.13), (2.16)-(2.18),

$$
\|W\|_{\mathcal{H}} \leq c\|F\|_{\mathcal{H}},
$$

i.e., the range of $A$ is all $\mathcal{H}$ and $A^{-1}$ is continuous, which proves $0 \in \varrho(A)$.

As a corollary (compare, e.g., Theorem 1.2.4 in [9]) we obtain that $-A$ generates a $C_{0}$-semigroup of contractions $\left\{e^{-t A}\right\}_{t \geq 0}$ on $\mathcal{H}$ and we solve $(2.7)$ as follows.

Theorem 2.2. The unique solution $V \in C_{0}([0, \infty), D(A)) \cap C^{1}([0, \infty), \mathcal{H})$ to $(2.7)$

$$
V_{t}(t)+A V(t)=0, \quad V(0)=V_{0} \in D(A)
$$

is given by

$$
V(t)=e^{-t A} V_{0}
$$

Higher regularity is obtained observing $V(t) \in D\left(A^{m}\right)$ if $V_{0} \in D\left(A^{m}\right), m \in \mathbb{N}$.

We observe that $A^{-1}$ is not compact in general: Let

$$
F_{n}:=\left(\begin{array}{c}
0 \\
0 \\
0 \\
\operatorname{rot} \psi_{n}
\end{array}\right), \quad \psi_{n} \in C^{1}(\bar{\Omega}), \quad\left\|\operatorname{rot} \psi_{n}\right\|:=1 .
$$

Let $A U_{n}=F_{n}$. Then we see that

$$
U_{n}=\left(\begin{array}{c}
0 \\
0 \\
0 \\
\tau_{0} \operatorname{rot} \psi_{n}
\end{array}\right)
$$

but $\left(U_{n}\right)_{n}$ does not have a convergent subsequence in general.

Purely oscillatory behavior is observed if there exist purely imaginary eigenvalues of $A$, because if

$$
A W=i \xi W, W \in D(A) \backslash\{0\}, \xi \in \mathbb{R} \backslash\{0\},
$$

then

$$
\forall t \geq 0:\left\|e^{-t A} W\right\|_{\mathcal{H}}=\left\|e^{-i \xi t} W\right\|_{\mathcal{H}}=\|W\|
$$

We remark that the spaces and the norms have been chosen such that $\frac{1}{2}\|V(t)\|_{\mathcal{H}}^{2}$ equals the encrgy of first order to be studied in the next sections. Hence (2.21) expresses 
conservation of energy for data $W$ satisfying (2.20), in particular no decay rate at all, in this case.

Let $W$ satisfy $(2.20)$. Then we conclude

$$
\beta \gamma\left\|W^{4}\right\|^{2}=\operatorname{Re}\left(i \xi\|W\|_{\mathcal{H}}^{2}\right)=0 .
$$

Hence

$$
W^{4}=0
$$

which implies

$$
\begin{gathered}
-W^{2}=i \xi W^{1}, \\
E W^{1}+\beta \nabla W^{3}=i \xi W^{2}, \\
\beta \operatorname{div} W^{2}=i \xi W^{3}, \\
\kappa \nabla W^{3}=0,
\end{gathered}
$$

from which we conclude successively

$$
W^{3}=0
$$

and

$$
\begin{gathered}
E W^{1}=|\xi|^{2} W^{1}, \quad W^{1} \in D(A), \\
\operatorname{div} W^{1}=0 .
\end{gathered}
$$

That is, there are purely imaginary eigenvalues if and only if the eigenvalue problem (2.22) has a solution satisfying side condition (2.23). This is the same situation as in classical thermoelasticity $\left(\tau_{0}=0\right.$; see $[3]$ or $\left.[12,5]\right)$ and leads to the conjecture that the asymptotic behavior in thermoclasticity with second sound is essentially the same as that in classical thermoelasticity, not worse in general. We shall prove in the next sections that this is true concerning exponential stability in symmetrical situations.

As is proved in [3] (compare also [12]), solutions to initial data essentially orthogonal to the eigenspace corresponding to purely imaginary eigenvalues tend to zero. In cases where $A^{-1}$ can be shown to be compact, for example in the radially symmetric case where in particular $\operatorname{rot} q=0$ and $\nu \times q=0$, the same behavior can also be proved; compare $[12,3]$. Here, the question of exponential stability for special cases is investigated, namely for those only known situations where exponential stability is known for the "more dissipative" system of classical thermoelasticity.

We remark that (2.22), (2.23) has or has no solutions depending on the domain; e.g., in balls there are solutions and hence an oscillating behavior of solutions is possible in general (see for instance $[12,5]$ ). Another comparison with the limit $\tau_{0} \rightarrow 0$ is given in the next section.

3. Exponential stability if $\operatorname{rot} u=\operatorname{rot} q=0$. In this section we assume a priori that the solution $(u, \theta, q)$ to $(1.1)-(1.5)$ additionally satisfies

$$
\begin{gathered}
\operatorname{rot} u=\operatorname{rot} q=0 \quad \text { in }[0, \infty) \times \Omega, \\
\nu \times q=0 \quad \text { in }[0, \infty) \times \partial \Omega,
\end{gathered}
$$


where the rotation is defined for $n=2$ as the scalar

$$
\operatorname{rot}\left(\begin{array}{l}
u^{1} \\
u^{2}
\end{array}\right):=\partial_{1} u^{2}-\partial_{2} u^{1},
$$

and $\nu=\nu(x)$ denotes the exterior normal vector in $x \in \partial \Omega$. If the rotation of a scalar field $f$ is defined in $\mathbb{R}^{2}$ as

$$
\operatorname{rot} f:=\left(\begin{array}{c}
\partial_{2} f \\
-\partial_{1} f
\end{array}\right)
$$

then the formula

$$
\Delta=\nabla \operatorname{div}-\operatorname{rot} \operatorname{rot}
$$

holds in two and three space dimensions; moreover, if $\operatorname{rot} u=0$ and $u \in\left(H^{2}(\Omega) \cap\right.$ $\left.H_{0}^{1}(\Omega)\right)^{n}$, then

$$
\nabla \operatorname{div} u=\Delta u \quad \text { and } \quad\|\nabla u\|=\|\operatorname{div} u\|
$$

and

$$
\mu \Delta+(\mu+\lambda) \nabla \operatorname{div} u=\alpha \Delta u
$$

where

$$
\alpha:=2 \mu+\lambda
$$

We remark that the condition $\operatorname{rot} q=0$ is compatible with classical thermoelasticity where $q=-\kappa \nabla \theta$, and not a serious restriction, while the condition $\operatorname{rot} u=0$ will be satisfied in the radially symmetric case. From Sec. 2 we know that we cannot expect a decay of solutions in general.

Let the energy terms of first and second order be defined as

$$
\begin{aligned}
E_{1}(t) & :=\frac{1}{2} \int_{\Omega}\left\{\kappa \delta\left|u_{t}\right|^{2}+\kappa \delta \alpha|\nabla u|^{2}+\kappa \beta|\theta|^{2}+\tau \beta \gamma|q|^{2}\right\}(t, x) d x \\
& \equiv E(t ; u, \theta, q), \\
E_{2}(t) & :=E\left(t ; u_{t}, \theta_{t}, q_{t}\right) .
\end{aligned}
$$

We shall prove the exponential decay of $E_{1}(t)+E_{2}(t)$ as $t \rightarrow \infty$. For this purpose we shall have to combine multiplier techniques and boundary control estimates used in [4] for classical thermoelasticity with those used in [13], the latter of course adapted to the multi-dimensional case requiring additional considerations.

TheOREM 3.1. Let $(u, \theta, q)$ be the solution to (1.1)-(1.5) satisfying (3.1), (3.2). Then the associated energy

$$
E(t):=E_{1}(t)+E_{2}(t)
$$

decays exponentially, i.e.,

$$
\exists d_{0}, C_{0}>0 \quad \forall t \geq 0: \quad E(t) \leq C_{0} e^{-d_{0} t} E(0) .
$$

Proof. One easily computes

$$
\begin{aligned}
& \frac{d}{d t} E_{1}(t)=-\beta \gamma\|q\|^{2}, \\
& \frac{d}{d t} E_{2}(t)=-\beta \gamma\left\|q_{t}\right\|^{2} .
\end{aligned}
$$

Of course, this corresponds to the dissipativity of the operator $-A$ in (2.8). 
The differential equation (1.3) yields

$$
\|\nabla \theta\|^{2} \leq \frac{2 \tau_{0}^{2}}{\kappa^{2}}\left\|q_{t}\right\|^{2}+\frac{2}{\kappa^{2}}\|q\|^{2} .
$$

Multiplying (1.1) by $\frac{1}{\alpha} \Delta u$--observing (3.3)-we get

$$
\begin{aligned}
\|\Delta u\|^{2} & =\frac{1}{\alpha}\left\langle u_{t t}, \Delta u\right\rangle+\frac{\beta}{\alpha}\langle\nabla \theta, \Delta u\rangle \\
& \leq-\frac{1}{\alpha} \frac{d}{d t}\left\langle\nabla u_{t}, \nabla u\right\rangle+\frac{1}{\alpha}\left\|\nabla u_{t}\right\|^{2}+\frac{3}{4} \frac{\beta^{2}}{\alpha^{2}}\|\nabla \theta\|^{2}+\frac{1}{3}\|\Delta u\|^{2}
\end{aligned}
$$

implying

$$
\frac{2}{3}\|\Delta u\|^{2}+\frac{1}{\alpha} \frac{d}{d t}\left\langle\nabla u_{t}, \nabla u\right\rangle \leq \frac{1}{\alpha}\left\|\nabla u_{t}\right\|^{2}+\frac{3}{4} \frac{\beta^{2}}{\alpha^{2}}\|\nabla \theta\|^{2} .
$$

Multiplying (1.2) by $\frac{3}{\alpha \delta} \operatorname{div} u_{t}$, we obtain

$$
\begin{aligned}
\frac{3}{\alpha}\left\|\operatorname{div} u_{t}\right\|^{2} & =-\frac{3 \gamma}{\alpha \delta}\left\langle\operatorname{div} q, \operatorname{div} u_{t}\right\rangle-\frac{3}{\alpha \delta}\left\langle\theta_{t}, \operatorname{div} u_{t}\right\rangle \\
& =\frac{3 \gamma}{\alpha \delta}\left\langle q, \nabla \operatorname{div} u_{t}\right\rangle-\frac{3 \gamma}{\alpha \delta}\left\langle\nu q, \operatorname{div} u_{t}\right\rangle_{\partial \Omega}+\frac{3}{\alpha \delta}\left\langle\nabla \theta_{t}, u_{t}\right\rangle,
\end{aligned}
$$

where $\langle\cdot, \cdot\rangle_{\partial s}$ denotes the $L^{2}(\partial \Omega)$-inner product with norm $\|\cdot\|_{\partial \Omega}$.

Thus, using the equations (1.1), (1.3) again,

$$
\begin{aligned}
\frac{3}{\alpha}\left\|\operatorname{div} u_{t}\right\|^{2}= & \frac{3 \gamma}{\alpha \delta} \frac{d}{d t}\langle q, \Delta u\rangle-\frac{3 \gamma}{\alpha \delta}\left\langle q_{t}, \Delta u\right\rangle+\frac{3 \gamma}{\alpha \delta} \frac{d}{d t}\left\langle\nabla \theta, u_{t}\right\rangle \\
& -\frac{3}{\alpha \delta}\left\langle\nabla \theta, u_{t t}\right\rangle-\frac{3 \gamma}{\alpha \delta}\left\langle\nu q, \operatorname{div} u_{t}\right\rangle_{\partial \Omega} \\
= & \frac{3 \gamma}{\alpha^{2} \delta} \frac{d}{d t}\left\langle q, u_{t t}\right\rangle+\frac{3 \beta \gamma}{\alpha^{2} \delta} \frac{d}{d t}\langle q, \nabla \theta\rangle-\frac{3 \gamma}{\alpha \delta}\left\langle q_{t}, \Delta u\right\rangle \\
& +\frac{3}{\alpha \delta} \frac{d}{d t}\left\langle\nabla \theta, u_{t}\right\rangle-\frac{3}{\delta}\langle\nabla \theta, \Delta u\rangle+\frac{3 \beta}{\alpha \delta}\|\nabla \theta\|^{2}-\frac{3 \gamma}{\alpha \delta}\left\langle\nu q, \operatorname{div} u_{t}\right\rangle_{\partial \Omega} \\
\leq & \frac{3 \gamma}{\alpha^{2} \delta} \frac{d}{d t}\left\langle q, u_{t t}\right\rangle-\frac{3 \beta \gamma \tau_{0}}{\alpha^{2} \delta \kappa} \frac{d}{d t}\left\langle q, q_{t}\right\rangle-\frac{3 \beta \gamma}{\alpha^{2} \delta \kappa} \frac{d}{d t}\|q\|^{2} \\
& +\frac{27 \gamma^{2}}{\alpha^{2} \delta^{2}}\left\|q_{t}\right\|^{2}+\frac{1}{12}\|\Delta u\|^{2}-\frac{3 \tau_{0}}{\alpha \delta \kappa} \frac{d}{d t}\left\langle q_{t}, u_{t}\right\rangle-\frac{3}{\alpha \delta \kappa} \frac{d}{d t}\left\langle q, u_{t}\right\rangle \\
& +\frac{27}{\delta^{2}}\|\nabla \theta\|^{2}+\frac{1}{12}\|\Delta u\|^{2}+\frac{3 \beta}{\alpha \delta}\|\nabla \theta\|^{2}-\frac{3 \gamma}{\alpha \delta}\left\langle\nu q, \operatorname{div} u_{t}\right\rangle_{\partial \Omega} .
\end{aligned}
$$

Combining (3.7) and (3.8) we obtain, observing (3.3),

$$
\begin{aligned}
\frac{2}{\alpha}\left\|\nabla u_{t}\right\|^{2}+\frac{1}{2}\|\Delta u\|^{2}+\frac{d}{d t} G_{1}(t) \leq & \left(\frac{3}{4} \frac{\beta^{2}}{\alpha^{2}}+\frac{27}{\delta^{2}}+\frac{3 \beta}{\alpha \delta}\right)\|\nabla \theta\|^{2} \\
& +\frac{27 \gamma^{2}}{\alpha^{2} \delta^{2}}\left\|q_{t}\right\|^{2}-\frac{3 \gamma}{\alpha \delta}\left\langle\nu q, \operatorname{div} u_{t}\right\rangle_{\partial \Omega},
\end{aligned}
$$

where

$$
\begin{aligned}
G_{1}(t):= & \frac{1}{\alpha}\left\langle\nabla u_{t}, \nabla u\right\rangle-\frac{3 \gamma}{\alpha^{2} \delta}\left\langle q, u_{t t}\right\rangle+\frac{3 \beta \gamma \tau_{0}}{\alpha^{2} \delta \kappa}\left\langle q, q_{t}\right\rangle+\frac{3 \beta \gamma}{\alpha^{2} \delta \kappa}\|q\|^{2} \\
& +\frac{3 \tau_{0}}{\alpha \delta \kappa}\left\langle q_{t} u_{t}\right\rangle+\frac{3}{\alpha \delta \kappa}\left\langle q, u_{t}\right\rangle .
\end{aligned}
$$


Using the first Poincaré estimate for $u_{t}$ and $\theta$, as well as (1.1) for $u_{t t}$, we obtain

$$
\left\|u_{t t}\right\|^{2}+\left\|u_{t}\right\|^{2}+\|\theta\|^{2} \leq c\left(\|\Delta u\|^{2}+\|\nabla \theta\|^{2}+\left\|\nabla u_{t}\right\|^{2}\right)
$$

where $c>0$ will again denote a constant that may take different values at different places, $c=c\left(\alpha, \beta, \gamma, \tau_{0}, \Omega\right)$.

Multiplying (1.1) by $u$, we get

$$
-\alpha\|\nabla u\|^{2}=\left\langle u_{t t}, u\right\rangle+\beta\langle\nabla \theta, u\rangle
$$

which gives, using Poincaré again,

$$
\frac{\alpha}{2}\|\nabla u\|^{2} \leq c\left(\left\|u_{t t}\right\|^{2}+\|\nabla \theta\|^{2}\right) .
$$

A multiplication of (1.2) by $\theta_{t}$ yields

$$
\begin{aligned}
\left\|\theta_{t}\right\|^{2} & =-\gamma\left\langle\operatorname{div} q, \theta_{t}\right\rangle-\delta\left\langle\operatorname{div} u_{t}, \theta_{t}\right\rangle \\
& \leq \gamma\left\langle q, \nabla \theta_{t}\right\rangle+\frac{\delta^{2}}{2}\left\|\operatorname{div} u_{t}\right\|^{2}+\frac{1}{2}\left\|\theta_{t}\right\|^{2} \\
& =\frac{d}{d t}\langle q, \nabla \theta\rangle-\gamma\left\langle q_{t}, \nabla \theta\right\rangle+\frac{\delta^{2}}{2}\left\|\operatorname{div} u_{t}\right\|^{2}+\frac{1}{2}\left\|\theta_{t}\right\|^{2}
\end{aligned}
$$

whence, for arbitrary $\tilde{\mu}>0$,

$$
\tilde{\mu}\left\|\theta_{t}\right\|^{2}-2 \tilde{\mu} \frac{d}{d t}\langle q, \nabla \theta\rangle \leq \tilde{\mu} \gamma\left\|q_{t}\right\|^{2}+\tilde{\mu} \gamma\|\nabla \theta\|^{2}+\delta^{2} \tilde{\mu}\left\|\operatorname{div} u_{t}\right\|^{2}
$$

follows, and $\tilde{\mu}$ will be determined later.

The boundary term appearing in (3.9) will be treated next, in various steps using boundary control estimates; compare [4] for the simpler situation in classical thermoelasticity.

$$
\left|\frac{3 \gamma}{\alpha \delta}\left\langle\nu q, \operatorname{div} u_{t}\right\rangle_{\partial \Omega}\right| \leq \frac{c_{1}}{\hat{\varepsilon}}\|\nu q\|_{\partial \Omega}^{2}+\hat{\varepsilon}\left\|\operatorname{div} u_{t}\right\|_{\partial \Omega}^{2}
$$

where $1>\hat{\varepsilon}>0$ is still arbitrary and will be determined later, and $c_{1}$ (similarly $c_{2}, c_{3}, \ldots$ ) denotes a fixed constant.

Let $\sigma \in\left(C^{1}(\bar{\Omega})\right)^{3}$ be such that $\sigma=\left(\sigma_{i}\right)_{i=1, \cdot, n}$ with $\sigma_{k}=\nu_{k}$ on $\partial \Omega$, and let $\partial_{k}:=\frac{\partial}{\partial x_{k}}$, $k=1, \cdot, n$.

Multiplying (1.2) by $-\frac{\beta}{\delta} \sigma_{k} \partial_{k} \theta_{t}$ (summation convention), we obtain

$$
\begin{aligned}
0= & -\frac{\beta}{\delta}\left\langle\theta_{t}, \sigma_{k} \partial_{k} \theta_{t}\right\rangle-\frac{\beta \gamma}{\delta}\left\langle\operatorname{div} q, \sigma_{k} \partial_{k} \theta_{t}\right\rangle-\beta\left\langle\operatorname{div} u_{t}, \sigma_{k} \partial_{k} \theta_{t}\right\rangle \\
= & -\frac{\beta}{\delta}\left\|\theta_{t}\right\|_{\partial \Omega}^{2}+\frac{\beta}{2 \delta}\left\langle(\operatorname{div} \sigma) \theta_{t}, \theta_{t}\right\rangle-\frac{\beta \gamma}{\delta} \frac{d}{d t}\left\langle\operatorname{div} q, \sigma_{k} \partial_{k} \theta\right\rangle+\frac{\beta \gamma}{\delta}\left\langle\operatorname{div} q_{t}, \sigma_{k} \partial_{k} \theta\right\rangle \\
& -\beta\left\langle\operatorname{div} u_{t}, \sigma_{k} \partial_{k} \theta_{t}\right\rangle \\
= & -\frac{\beta}{\delta}\left\|\theta_{t}\right\|_{\partial \Omega}^{t}+\frac{\beta}{2 \delta}\left\langle(\operatorname{div} \sigma) \theta_{t}, \theta_{t}\right\rangle-\frac{\beta \gamma}{\delta} \frac{d}{d t}\left\langle\operatorname{div} q, \sigma_{k} \partial_{k} \theta\right\rangle \\
& -\frac{\beta \gamma}{\tau_{0} \delta}\left\langle\operatorname{div} q, \sigma_{k} \partial_{k} \theta\right\rangle-\frac{\beta \gamma \kappa}{\tau_{0} \delta}\left\langle\Delta \theta, \sigma_{k} \partial_{k} \theta\right\rangle-\beta\left\langle\operatorname{div} u_{t}, \sigma_{k} \partial_{k} \theta_{t}\right\rangle .
\end{aligned}
$$


Since

$$
\begin{aligned}
\left\langle\Delta \theta, \sigma_{k} \partial_{k} \theta\right\rangle & =\left\langle\nu \nabla \theta, \nu_{k} \partial_{k} \theta\right\rangle_{\partial \Omega}-\left\langle\nabla \theta,\left(\nabla \sigma_{k}\right) \partial_{k} \theta\right\rangle-\left\langle\nabla \theta, \sigma_{k} \partial_{k} \nabla \theta\right\rangle \\
& =\left\|\frac{\partial \theta}{\partial \nu}\right\|_{\partial \Omega}^{2}-\left\langle\nabla \theta,\left(\nabla \sigma_{k}\right) \partial_{k} \theta\right\rangle-\frac{1}{2}\|\nabla \theta\|_{\partial \Omega}^{2}+\frac{1}{2}\langle(\operatorname{div} \sigma) \nabla \theta, \nabla \theta\rangle \\
& =\frac{1}{2}\left\|\frac{\partial \theta}{\partial \nu}\right\|_{\partial \Omega}^{2}-\left\langle\nabla \theta,\left(\nabla \sigma_{k}\right) \partial_{k} \theta\right\rangle+\frac{1}{2}\langle(\operatorname{div} \sigma) \nabla \theta, \nabla \theta\rangle,
\end{aligned}
$$

where we used the boundary condition $\theta_{\mid \partial \Omega}=0$ to conclude that $\nabla \theta=\frac{\partial \theta}{\partial \nu} \cdot \nu$, we get from $(3.15)$

$$
\begin{aligned}
0= & \frac{2 \beta}{\delta}\left\|\theta_{t}\right\|_{\partial \Omega}^{2}-\frac{\beta}{\delta}\left\langle(\operatorname{div} \sigma) \theta_{t}, \theta_{t}\right\rangle+\frac{2 \beta \gamma}{\delta} \frac{d}{d t}\left\langle\operatorname{div} q, \sigma_{k} \partial_{k} \theta\right\rangle \\
& +\frac{2 \beta \gamma}{\tau_{0} \delta}\left\langle\operatorname{div} q, \sigma_{k} \partial_{k} \theta\right\rangle+\frac{\beta \gamma \kappa}{\tau_{0} \delta}\left\|\frac{\partial \theta}{\partial \nu}\right\|_{\partial \Omega}^{2}-\frac{2 \beta \gamma \kappa}{\tau_{0} \delta}\left\langle\nabla \theta,\left(\nabla \sigma_{k}\right) \partial_{k} \theta\right\rangle \\
& -\frac{\beta \gamma \kappa}{\tau_{0} \delta}\langle(\operatorname{div} \sigma) \nabla \theta, \nabla \theta\rangle+2 \beta\left\langle\operatorname{div} u_{t}, \sigma_{k} \partial_{k} \theta_{t}\right\rangle .
\end{aligned}
$$

On the other hand we obtain in the same way, differentiating (1.1) with respect to $t$ and multiplying by $\sigma_{k} \partial_{k} u_{t}$-actually an application of Lemma 2.3 from [4]--

$$
\begin{aligned}
0= & \left\|\frac{\partial u_{t}}{\partial \nu}\right\|_{\partial \Omega}^{2}+(\mu+\lambda)\left\|\operatorname{div} u_{t}\right\|_{\partial \Omega}^{2}-2 \frac{d}{d t}\left\langle u_{t t}, \sigma_{k} \partial_{k} u_{t}\right\rangle \\
& -\left\langle(\operatorname{div} \sigma) u_{t t}, u_{t t}\right\rangle-2 \mu\left\langle\partial_{j} u_{t}^{i},\left(\partial_{j} \sigma_{k}\right) \partial_{k} u_{t}^{i}\right\rangle \\
& +\mu\left\langle(\operatorname{div} \sigma) \nabla u_{t}, \nabla u_{t}\right\rangle-2(\mu+\lambda)\left\langle\operatorname{div} u_{t},\left(\nabla \sigma_{k}\right) \partial_{k} u_{t}\right\rangle \\
& +(\mu+\lambda)\left\langle(\operatorname{div} \sigma) \operatorname{div} u_{t}, \operatorname{div} u_{t}\right\rangle-2 \beta\left\langle\nabla \theta_{t}, \sigma_{k} \partial_{k} u_{t}\right\rangle .
\end{aligned}
$$

Moreover, we have

$$
\begin{aligned}
\operatorname{Re}\left\langle\operatorname{div} u_{t}, \sigma_{k} \partial_{k} \theta_{k}\right\rangle= & -\operatorname{Re}\left\langle\partial_{k} \partial_{m} u_{t}^{m}, \sigma_{k} \theta_{t}\right\rangle-\operatorname{Re}\left\langle\partial_{m} u_{t}^{m},\left(\partial_{k} \sigma_{k}\right) \theta_{t}\right\rangle \\
= & -\operatorname{Re}\left\langle\theta_{t}, \sigma_{k} \partial_{k} \partial_{m} u_{t}^{m}\right\rangle-k\left\langle\theta_{t}\left(\partial_{m} \sigma_{k}\right) \partial_{k} u_{t}^{m}\right\rangle \\
& +\operatorname{Re}\left\langle\theta_{t},\left(\partial_{m} \sigma_{k}\right) \partial_{k} u_{t}^{m}-\left(\partial_{k} \sigma_{k}\right) \partial_{m} u_{t}^{m}\right\rangle \\
= & \operatorname{Re}\left\langle\partial_{m} \theta_{t}, \sigma_{k} \partial_{k} u_{t}^{m}\right\rangle+\operatorname{Re}\left\langle\theta_{t},\left(\partial_{m} \sigma_{k}\right) \partial_{k} u_{t}^{m}-\left(\partial_{t} \sigma_{k}\right) \partial_{m} u_{t}^{m}\right\rangle \\
= & \operatorname{Re}\left\langle\nabla \theta_{t}, \sigma_{k} \partial_{k} u_{t}\right\rangle+\operatorname{Re}\left\langle\theta_{t},\left(\nabla \sigma_{k}\right) \partial_{k} u_{t}-(\operatorname{div} \sigma) \operatorname{div} u_{t}\right\rangle
\end{aligned}
$$

Adding (3.16) and (3.17), taking real parts and observing (3.18), we obtain

$$
\begin{gathered}
\frac{2 \beta}{\delta}\left\|\theta_{t}\right\|_{\partial \Omega}^{2}+\frac{\beta \gamma \kappa}{\tau_{0} \delta}\left\|\frac{\partial \theta}{\partial \nu}\right\|_{\partial \Omega}^{2}+\left\|\frac{\partial u_{t}}{\partial \nu}\right\|_{\partial \Omega}^{2}+(\mu+\lambda)\left\|\operatorname{div} u_{t}\right\|_{\partial \Omega}^{2} \\
+\frac{d}{d t} \operatorname{Re}\left(\frac{2 \beta \gamma}{\delta}\left\langle\operatorname{div} q, \sigma_{k} \partial_{k} \theta\right\rangle-2\left\langle u_{t t}, \sigma_{k} \partial_{k} u_{t}\right\rangle\right) \\
\leq c_{2}\left(\left\|\theta_{t}\right\|^{2}+\|\nabla \theta\|^{2}+\left\|\nabla u_{t}\right\|^{2}+\|\Delta u\|^{2}\right) .
\end{gathered}
$$

Until now we only used $\operatorname{rot} u=0$ from the assumptions (3.1), (3.2). In the next step we also exploit $\operatorname{rot} q=0$ and $\nu \times q=0$ on $\partial \Omega$.

Multiplying (1.2) by $\sigma q$ yields

$$
\tau_{0}\left\langle\theta_{t}, \sigma q\right\rangle+\gamma\langle\operatorname{div} q, \sigma q\rangle+\delta\left\langle\operatorname{div} u_{t}, \sigma q\right\rangle=0
$$


with

$$
\left|\tau_{0}\left\langle\theta_{t}, \sigma q\right\rangle\right| \leq \tilde{\varepsilon}\left\|\theta_{t}\right\|^{2}+\frac{c_{3}}{\tilde{\varepsilon}}\|q\|^{2}
$$

for some $\tilde{\varepsilon}>0$ to be determined later.

$$
\begin{gathered}
\left|\delta\left\langle\operatorname{div} u_{t}, \sigma q\right\rangle\right| \leq \tilde{\varepsilon}\left\|\operatorname{div} u_{t}\right\|^{2}+\left.\frac{c_{4}}{\tilde{\varepsilon}}|q|\right|^{2}, \\
\langle\operatorname{div} q, \sigma q\rangle=\left\langle\partial_{j} q_{j}, \sigma_{k} q_{k}\right\rangle=\left\langle\nu_{j} q_{j}, \sigma_{k} q_{k}\right\rangle_{\partial \Omega}-\left\langle q_{j},\left(\partial_{j} \sigma_{k}\right) g_{k}\right\rangle-\left\langle q_{j}, \sigma_{k} \partial_{j} g_{k}\right\rangle \\
=\|\nu q\|_{\partial \Omega}^{2}-\left\langle q_{j},\left(\partial_{j} \sigma_{k}\right) q_{k}\right\rangle-\left\langle q_{j}, \sigma_{k} \partial_{k} q_{j}\right\rangle \\
=\|\nu q\|^{2}-\left\langle q_{j},\left(\partial_{j} \sigma_{k}\right) q_{k}\right\rangle-\frac{1}{2}\|q\|_{\partial \Omega}^{2}+\frac{1}{2}\langle q,(\operatorname{div} \sigma) q\rangle \\
=\frac{1}{2}\|\nu q\|^{2}-\left\langle q_{j},\left(\partial_{j} \sigma_{k}\right) q_{k}\right\rangle+\frac{1}{2}\langle q,(\operatorname{div} \sigma) q\rangle .
\end{gathered}
$$

Combining (3.20)-(3.23), we conclude ( $\tilde{\varepsilon}$ small):

$$
\|\nu q\|^{2} \leq \tilde{\varepsilon}\left(\left\|\theta_{t}\right\|^{2}+\left\|\operatorname{div} u_{t}\right\|^{2}\right)+\frac{c_{5}}{\tilde{\varepsilon}}\|q\|^{2}
$$

From (3.9), (3.13), (3.14), (3.19) (multiplied by $\left.\frac{\hat{\varepsilon}}{\mu+\lambda}\right)$, (3.24) follows for sufficiently small $\tilde{\mu}, \hat{\varepsilon}, \tilde{\varepsilon}$ :

$$
\frac{1}{\alpha}\left\|\nabla u_{t}\right\|^{2}+\frac{1}{4}\|\Delta u\|^{2}+c_{6}\left\|\theta_{t}\right\|^{2}+\frac{d}{d t} H(t) \leq c_{7}\left(\left\|q_{t}\right\|^{2}+\|q\|^{2}\right),
$$

where (compare $(3.10)$ for $\left.G_{1}(t)\right)$

$$
H(t):=G_{1}(t)+\frac{\hat{\varepsilon}}{\mu+\lambda} \operatorname{Re}\left(\frac{2 \beta \gamma}{\delta}\left\langle\operatorname{div} q, \sigma_{k} \partial_{k} \theta\right\rangle-2\left\langle u_{t}, \sigma_{k} \partial_{k} u_{t}\right\rangle\right)-2 \tilde{\mu}\langle q, \nabla \theta\rangle .
$$

A suitable Lyapunov function $F$ is defined by

$$
F(t):=\frac{1}{\varepsilon}\left(E_{1}(t)+E_{2}(t)\right)+H(t),
$$

where $\varepsilon>0$.

Combining (3.3), (3.5), (3.11), (3.12), and (3.25), we see that $F$ satisfies, for sufficiently small $\varepsilon$,

$$
\frac{d}{d t} F(t) \leq-d_{1}\left(E_{1}(t)+E_{2}(t)\right)
$$

for some $d_{1}>0$. On the other hand ( $\varepsilon$ small enough),

$$
\exists C_{1}, C_{2}>0 \quad \forall t \geq 0: C_{1} E(t) \leq F(t) \leq C_{2} E(t),
$$

with

$$
E(t)=E_{1}(t)+E_{2}(t) .
$$

Hence, we conclude from (3.27),

$$
\frac{d}{d t} F(t) \leq-d_{0} F(t)
$$

with

$$
d_{0}:=\frac{d_{1}}{C_{2}}
$$

whence

$$
F(t) \leq e^{-d_{0} t} F(0)
$$


follows. Using (3.28) again, we conclude

$$
E(t) \leq C_{0} e^{-d_{0} t} E(0)
$$

with

$$
C_{0}:=\frac{C_{2}}{C_{1}}
$$

Q.E.D.

\section{Remarks.}

(1) The conditions (3.1), (3.2) will be satisfied in the radially symmetric case; see Sec. 4. The conditions on $q$, i.e., rot $q=0$ and $\nu \times q=0$ on $\partial \Omega$ are not very restrictive, since for $q_{0}$ with $\operatorname{rot} q_{0}=0$ and $\nu \times q_{0}=0$ on $\partial \Omega$, it follows from the differential equation (1.3). It is not known whether there are other applications.

(2) The constants $d_{0}$ and $C_{0}$ in Theorem 3.1 depend on $\Gamma:=\left(\alpha, \beta, \gamma, \delta, \tau_{0}\right)$ and on $\Omega$, where the latter dependence is given through the constant $C_{p}$ in the first Poincaré estimate for $v$ in $H_{0}^{1}(\Omega)$,

$$
\|v\| \leq C_{p}\|\nabla v\|
$$

and through the estimates on $\sigma, \nabla \sigma$. The dependence on $\Gamma$ could be described explicitly. Bounds for $d_{0}$ calculated in [13] in one dimension indicate a small decay rate.

(3) Using properties of the generator of the semigroup $A$, it could also be shown that the first energy $E_{1}$ alone tends to zero uniformly; compare [19, p. 64]. The estimates here allow to estimate the constants; see Remark 2.

As in one dimension [13], it can be shown that the energy of the difference of the solution $\left(u^{\tau_{0}}, \theta^{\tau_{0}}, q^{\tau_{0}}\right)$ to $(1.1)(1.5)$ and the solution $(\tilde{u}, \tilde{\theta}, \tilde{q})$ to the corresponding system with $\tau_{0}=0$ vanishes of order $\tau_{0}^{2}$, provided the compatibility condition

$$
q(0, \cdot)=-\kappa \nabla \tilde{\theta}(0, \cdot)
$$

holds. If $E_{1}^{\tau_{0}}$ denotes the first-order energy for the difference $\left(u^{\tau_{0}}-\tilde{u}, \theta^{\tau_{0}}-\tilde{\theta}, q^{\tau_{0}}-\tilde{q}\right) \equiv$ $(v, \varrho, z)$, then

$$
\frac{d}{d t} E_{1}^{\tau_{0}}(t)=-\beta \gamma\|z\|^{2}-\tau_{0} \beta \gamma \kappa\left\langle\nabla \tilde{\theta}_{t}, z\right\rangle
$$

implying

$$
E_{1}^{\tau_{0}}(t) \leq \tau_{0}^{2} \frac{\beta \gamma \kappa^{2}}{2} \int_{0}^{t}\left\|\nabla \tilde{\theta}_{t}(s, \cdot)\right\|^{2} d s,
$$

which holds in general, i.e., without assuming (3.1), (3.2).

Under the assumptions (3.1), (3.2) we have from [4]

$$
\int_{0}^{\infty}\left\|\nabla \tilde{\theta}_{t}(s, \cdot)\right\|^{2} d s<\infty
$$

implying

$$
\exists C>0 \quad \forall t \geq 0: E_{1}^{\tau_{0}}(t) \leq C \tau_{0}^{2} .
$$


4. Radial symmetry. Having proved Theorem 3.1 it follows now as in classical thermoelasticity $\left(\tau_{0}=0\right)$, see [4], the exponential stability for radially symmetric domains $\Omega$ and radially symmetric data. We recall that in a ball $\Omega$ there are-nonradially symmetric-data for which the energy $E(t)$ does not decay at all; compare Sec. 1 . We summarize the considerations from [4] carried over to our case $\tau_{0} \neq 0$ :

Let $\Omega$ be a smoothly bounded, radially symmetrical domain; i.e.,

$$
x \in \Omega \Rightarrow \forall R \in O(2) \quad(\text { if } n=2) \text { resp. } S O(3) \quad \text { (if } n=3): R x \in \Omega,
$$

where $(S) O$ denotes the (special) orthogonal group. A vector field $v$ and a function $\psi$ from $\Omega$ to $\mathbb{R}^{n}$ and $\mathbb{R}$, respectively, are called radially symmetric if

$$
\begin{array}{rll}
\forall R \in O(2) \quad(\text { if } n=2) \text { or } S O(3) \quad \text { (if } n=3) & \forall x \in \Omega: v(R x)=R v(x) \\
& \text { and } \quad \psi(R x)=\psi(x), \text { resp. }
\end{array}
$$

$v$ and $\psi$ are radially symmetric if and only if there are $\chi:[0, \infty) \rightarrow \mathbb{R}$ and $\zeta:[0, \infty)$ such that for $x \in \Omega$,

$$
v(x)=x \chi(|x|), \quad \psi(x)=\zeta(|x|) .
$$

Any radially symmetric vector field $v$ satisfies

$$
\operatorname{rot} v=0 \text { in } \Omega, \quad \nu \times v=0 \text { on } \partial \Omega .
$$

If the data $u_{0}, u_{1}, \theta_{0}, q_{0}$ in (1.4) are radially symmetric, then the solution $(u, \theta, q)$ to (1.1)-(1.5) is radially symmetric for all times. This can be seen taking for $R \in O(2)$ resp. $S O(3)\left(R^{t}\right.$ : transposed matrix)

$$
v(t, x):=R^{t} u(t, R x), \varrho(t, x):=\theta(t, R x), h(t, x):=R^{t} q(t, R x) .
$$

Then $(v, \varrho, h)$ also satisfies $(1.1)-(1.5)$; hence $(v, \varrho, h)=(u, \theta, q)$, implying the radial symmetry. In particular, $u$ and $q$ satisfy (4.1); hence (3.1), (3.2) is satisfied, and we can apply Theorem 3.1 .

TheOREM 4.1. Let $\Omega$ be radially symmetric and let the initial data $u_{0}, u_{1}, \theta_{0}, q_{0}$ be radially symmetric. Then the energy $E(t)=E_{1}(t)+E_{2}(t)$ associated to the solution $(u, \theta, q)$ if (1.1)-(1.4) decays exponentially; i.e.,

$$
\exists d_{0}, C_{0}>0 \quad \forall t \geq 0, \quad E(t) \leq C_{0} e^{-d_{0} t} E(0) .
$$

Acknowledgement. The author thanks the reviewer for his suggestions that led to an improvement of the presentation of the results.

\section{REFERENCES}

[1] D. E. Carlson, Linear thermoelasticity, Handbuch der Physik VIa/2 (1972), 297-346

[2] D. S. Chandrasekharaiah, Hyperbolic thermoelasticity: a review of recent literature, Appl. Mech. Rev. 51 (1998), 705-729

[3] C. M. Dafermos, On the existence and the asymptotic stability of solutions to the equations of linear thermoelasticity, Arch. Rational Mech. Anal. 29 (1968), 241-271

[4] S. Jiang, J. E. Muñoz Rivera, and R. Racke, Asymptotic stability and global existence in thermoelasticity with symmetry, Quart. Appl. Math. 56 (1998), 259-275

[5] S. Jiang and R. Racke, Evolution equations in thermoelasticity, $\pi$ Monographs Surveys Pure Appl. Math. 112, Chapman \& Hall/CRC, Boca Raton (2000)

[6] H. Koch, Slow decay in linear thermoelasticity, Quart. Appl. Math. 58 (2000), 601-612 
[7] G. Lebeau and E. Zuazua, Decay rates for the three-dimensional linear system of thermoclasticity, Arch. Rational Mech. Anal. 148 (1999), 179-231

[8] R. Leis, Initial boundary value problems in mathematical physics, B. G. Teubner-Verlag. Stuttgart; John Wiley \& Sons, Chichester (1986)

[9] Z. Liu and S. Zheng, Semigroups associated with dissipative systems, Chapman \& Hall/CRC Res. Notes Math. 398 (1999)

[10] H. W. Lord and Y. Shulman, A generalized dynamical theory of thermoelasticity, J. Mech. Phys. Solids 15 (1967), 299-309

[11] M. Mosbacher, V. Dobler, H.-J. Münzer, J. Zimmermann, J. Solis, J. Boneberg, and P. Leiderer, Optical field enhancement effects in laser assisted particle removal, Appl. Phys. A 72 (1991), 41-44

[12] R. Racke, On the time-asymptotic behaviour of solutions in thermoclasticity, Proc. Roy. Soc. Edinburgh 107A (1987), 289-298.

[13] R. Racke, Thermoelasticity with second sound-Exponential stability in linear and nonlinear 1-d, Konstanzer Schriften Math. Inf. 141 (2001)

[14] T. Sabir Öncü and T. Bryant Moodie, On the constitutive relations for second sound in elastic solids, Arch. Rational Mech. Anal. 121 (1992), $87-99$

[15] H. Sherief, On uniqueness and stability in generalized thermoelasticity, Quart. Appl. Math. 44 (1987), 773-778

[16] K. K. Tamma and R. R. Namburu, Computational approaches with applications to nonclassical and classical thermomechanical problems, Appl. Mech. Rev. 50 (1997), 514-551

[17] M. A. Tarabek, On the existence of smooth solutions in one-dimensional nonlinear thermoelasticity with second sound, Quart. Appl. Math. 50 (1992), 727-742

[18] X. Wang and X. Xu, Thermoelastic wave induced by pulsed laser heating, Appl. Phys. A (to appear). DOI: $10.1007 / \mathrm{s} 003390000593$

[19] S. Zheng, Nonlinear parabolic equations and hyperbolic-parabolic coupled systems, Pitman Monographs Surv. Pure Appl. Math. 76, Longman; John Wiley \& Sons, New York (1995) 